\title{
Myocardial Infarction Complicating Vasospastic Angina in a Female Patient-Case Report
}

\author{
Serigne Cheikh Tidiane Ndao', Mame Madjiguene Ka1, Khadidiatou Dia1, Amer Zabalawi2, \\ Régis Delaunay², Laurent Payot ${ }^{2}$, Joseph Salvador Mingou1, Mouhamed Chérif Mboup1, \\ Pape Diadie Fall 1
}

${ }^{1}$ Department of Cardiology, Hospital Principal Dakar, Dakar, Senegal

${ }^{2}$ Department of Cardiology, Yves Le Foll General Hospital, Saint-Brieuc, France

Email: sctndao@gmail.com

How to cite this paper: Ndao, S.C.T., Ka, M.M., Dia, K., Zabalawi, A., Delaunay, R., Payot, L., Mingou, J.S., Mboup, M.C. and Fall, P.D. (2020) Myocardial Infarction Complicating Vasospastic Angina in a Female Patient-Case Report. World Journal of Cardiovascular Diseases, 10, 155-161. https://doi.org/10.4236/wjcd.2020.103016

Received: February 27, 2020

Accepted: March 27, 2020

Published: March 30, 2020

Copyright $\odot 2020$ by author(s) and Scientific Research Publishing Inc. This work is licensed under the Creative Commons Attribution International License (CC BY 4.0).

http://creativecommons.org/licenses/by/4.0/

\begin{abstract}
Coronary artery vasospasm is an infrequent cause of acute coronary syndrome (ACS). A 50-year-old female developed persistent chest discomfort related to anterior STEMI. After an unsuccessful thrombolysis attempt, due to delay concern, the patient was then sent to the catheterization laboratory for rescue PCI. The coronary angiogram revealed a TIMI 3 flow proximal LAD significant stenosis with spastic appearance of the coronary arteries which has improved after intracoronary nitrates administration. Optical Coherence Tomography (OCT) excluded coronary artery dissection and haematoma also revealed thrombus on moderate atherosclerotic plaque. The patient was then pain-free; she was managed medically and discharged after few days of monitoring. Three weeks later, she developed transient chest pain with ST segment elevation while cycling during rehabilitation program. Provocation test by Ergonovine was positive with complete occlusion of proximal RCA (right coronary artery) complicated by transient complete heart block and shock. She was stabilized with $7 \mathrm{mg}$ of intracoronary nitrates injection. She was discharged after close monitoring with calcium channel blocker and tobacco cessation. ACS may complicate prolonged coronary artery vasospasm making the diagnosis challenging. OCT may help understand ACS mechanism and exclude spontaneous coronary artery dissection. Provocation test is crucial for confirmation.
\end{abstract}

\section{Keywords}

Vasospastic Angina, Acute Coronary Syndrome, Female Patient

\section{Background}

Vasospastic angina (VSA) known as Prinzmetal's angina is characterized by tran- 
sient myocardial ischemia. Precise prevalence remains to be determined; however, it seems to be more frequent in younger patients and females [1]. Clinical features include episodes of rest angina that promptly respond to short-acting nitrates with transient ST-segment elevation on the electrocardiogram occurring typically at night [2]. Prolonged coronary spasm may lead to authentic acute myocardial infarction in patients with or without atherosclerosis. Furthermore, VSA can be associated with different cardiac conditions such as stable angina, acute coronary syndrome, life-threatening arrhythmic events and sudden cardiac death [3] [4]. We report a case of anterior myocardial infarction due to vasospastic angina in a female patient confirmed ex post facto.

\section{Case Presentation}

A 50-year-old female with a single risk factor of active smoking was admitted to our Emergency Department for persistent chest discomfort lasting for 3 hours. She reported one month before, a similar episode at rest lasting for 30 minutes with spontaneous resolution. Her past medical history is only significant for depression and epilepsy.

On admission, her electrocardiogram (ECG) showed ST segment elevation in Leads V1 to 6, AVL and D1 with reciprocal ST depression in the inferior leads. Due to a long-time delay to the Catheterization laboratory (Cath lab), she received thrombolytic therapy without success since the ST segment elevation and the chest pain remain unchanged. She was immediately transferred to the Cath lab.

The coronary angiogram (CA) revealed a proximal LAD smooth and significant long stenosis with TIMI (Thrombolysis in Myocardial Infarction) 3 flow (Figure 1(a)). Furthermore, coronary arteries had a spastic appearance which was more obvious after OCT run in the LAD (Figure 1(b)). Intracoronary nitrates improved the coronary flow and deleted the spastic appearance (Figure 1(d)).

Intracoronary imaging by optical coherence tomography (OCT) to exclude SCAD (Spontaneous Coronary Artery Dissection) showed an important thrombus burden laying on a moderate atherosclerotic plaque. There was no evidence of coronary wall haematoma and dissection or plaque erosion or rupture.

She was then pain-free with partial resolution of the ST segment elevation before her admission to our CCU (Coronary Care Unit) for monitoring. She was treated with double antiplatelet (Aspirin, Ticagrelor), statins, PPI (Proton Pump Inhibitor) and unfractionated heparin. Echocardiogram revealed severe left ventricular dysfunction (Ejection fraction 35\% with wall abnormalities: akinetic apex and hypokinetic anteroseptal wall). Low dose ACE inhibitor and betablocker were introduced. The evolution in the CCU was favorable.

A week later, a planned coronary angiogram showed a similar finding without worsening of the LAD lesion (Figure 2), anticoagulation was stopped.

The patient was then discharged with a prescription of cardiac rehabilitation program. 


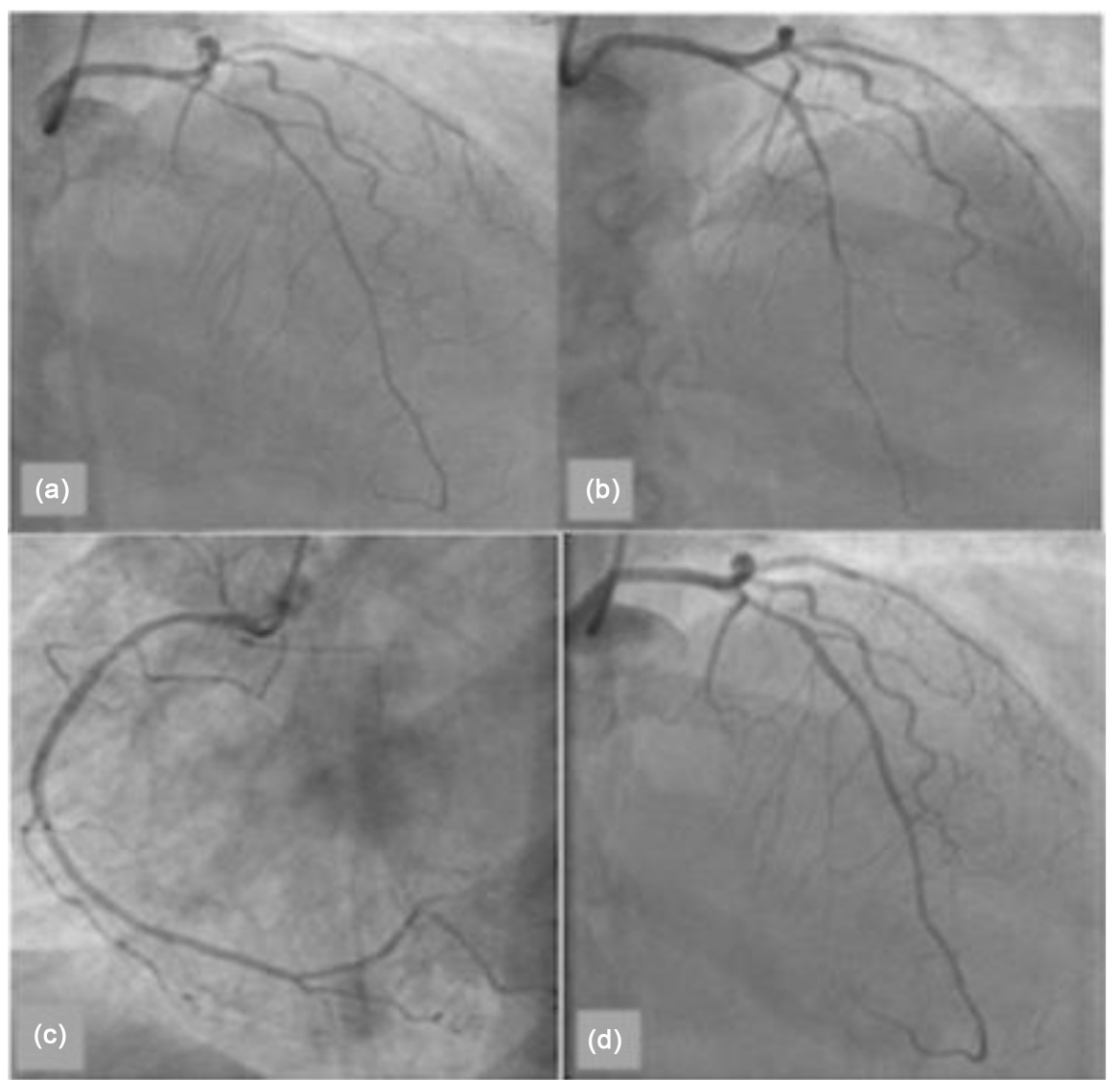

Figure 1. First hospitalization with acute coronary syndrome presentation. Significant stenosis in the proximal LAD (a); spastic LAD after OCT run (b); normal RCA (c). LAD after nitrates (d). LAD: left anterior descending coronary artery; RCA: right coronary artery.

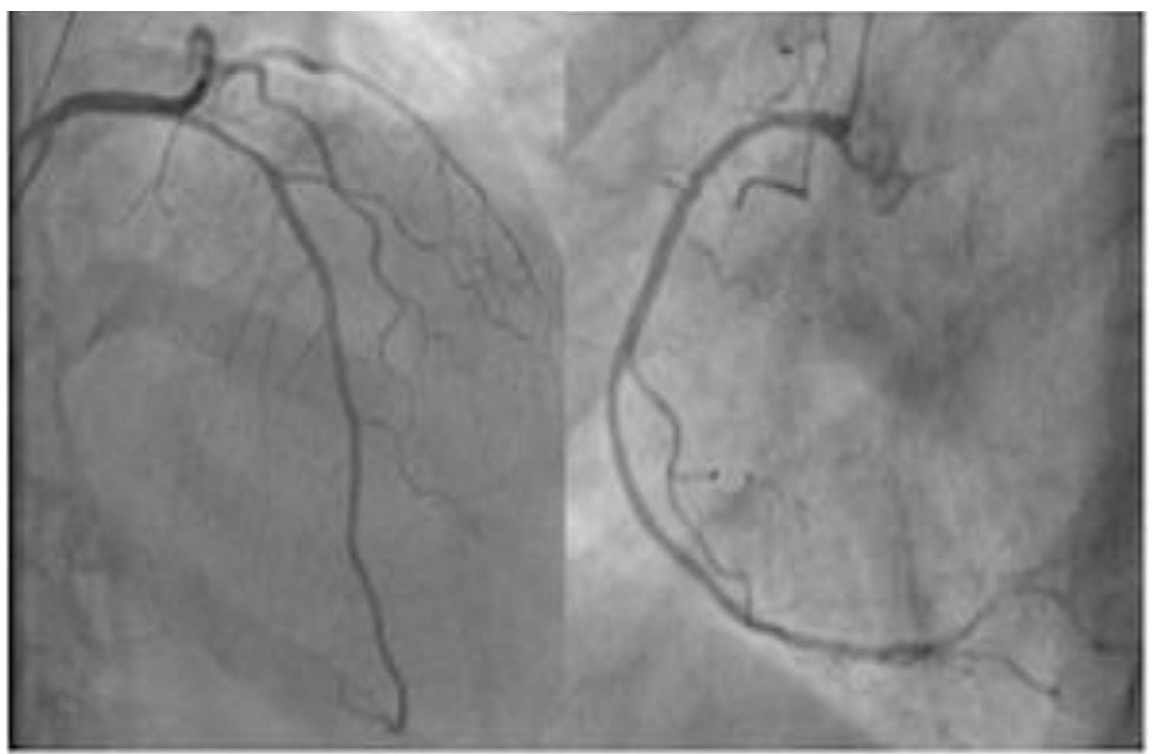

(a)

(b)

Figure 2. First hospitalization with acute coronary syndrome presentation. Coronary angiogram after one week. LAD (a); RCA (b). 
The latter involves 3 keys elements:

- Exercise training to improve cardiovascular fitness using a treadmill, bicycle or by walking;

- Education about atheromatous disease, conditions such as high blood pressure, diabetes, high cholesterol and lifestyle changes such as eating a hearthealthy diet, keeping a healthy weight and quitting smoking;

- Follow-up, usually organized with the patient's cardiologist.

However, three weeks later she experienced, during exercise test in rehabilitation program, transient chest pain with ST segment elevation. The CA findings were identical to the previous one. Provocation test by intracoronary administration of Ergonovine to exclude coronary vasospasm was positive with:

- Recurrent constrictive chest pain,

- ST segment elevation in the inferior and anterior leads and reciprocal ST segment depression in the lateral leads. Major conduction troubles were noted with high degree AV block then transient complete AV block before brief asystole (Figure 3(a') and Figure 3(b'));

- The right coronary artery was completely occluded in its proximal portion (Figure 3(a) and Figure 3(b)) with hemodynamic compromise.

The coronary artery was re-opened with $7 \mathrm{mg}$ intracoronary nitrate injection and $1 \mathrm{mg}$ intravenous atropine administration followed by resolution of pain and ECG abnormalities as well as hemodynamic stability.

The patient was then admitted at the CCU for monitoring with continuous Nitroglycerine infusion and discontinuity of betablocker. She was discharged with a treatment of aspirin, statin, ACE (Angiotensin-converting enzyme) inhibitor and Verapamil, after $48 \mathrm{~h}$ of surveillance at the CCU. She received advices and measures for definite and complete smoking cessation by an addiction specialist.

The follow-up was planned for every 6 months with exercise test, ECG and echocardiogram and clinic assessment.

Provocation Test: After 4 min occlusion of the distal segment of RCA1 (a) with high degree atrio-ventricular (AV) bloc (a'). At 5 minutes, occlusion of the proximal part (b) with asystole (b'). Normal RCA after $7 \mathrm{mg}$ of intracoronary Nitroglycerine (c).

\section{Discussion}

The diagnosis of vasospastic angina complicated by myocardial infarction can be challenging. Furthermore, the coronary angiographic pattern of our patient matched the type 2 of SCAD (Spontaneous Coronary Artery Dissection) described by SAW [5]. A young female patient with single risk factor of cardiovascular disease with ACS presentation should prompt us to rule out SCAD diagnosis. It accounts for approximately one third of young women ( $<60$ years) and with one or less cardiovascular risk factor presenting with ACS [6]. In this particular setting with morphologic ambiguity, OCT which provides high-resolution 


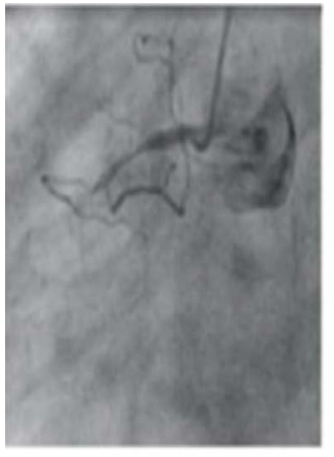

(a)

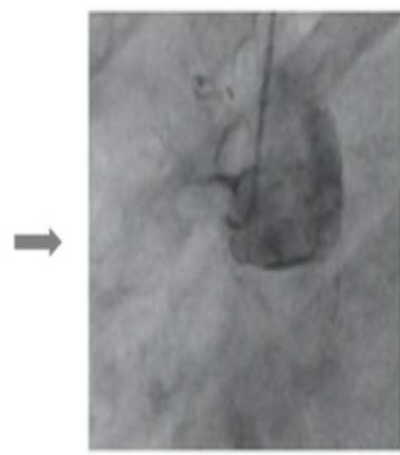

(b)

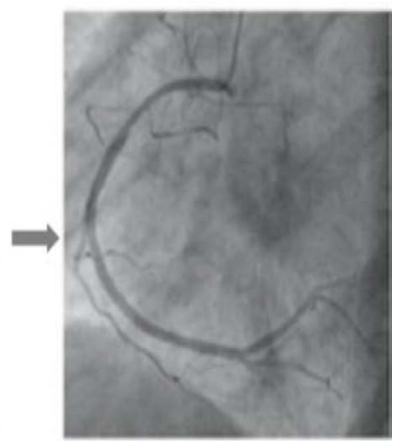

(c)

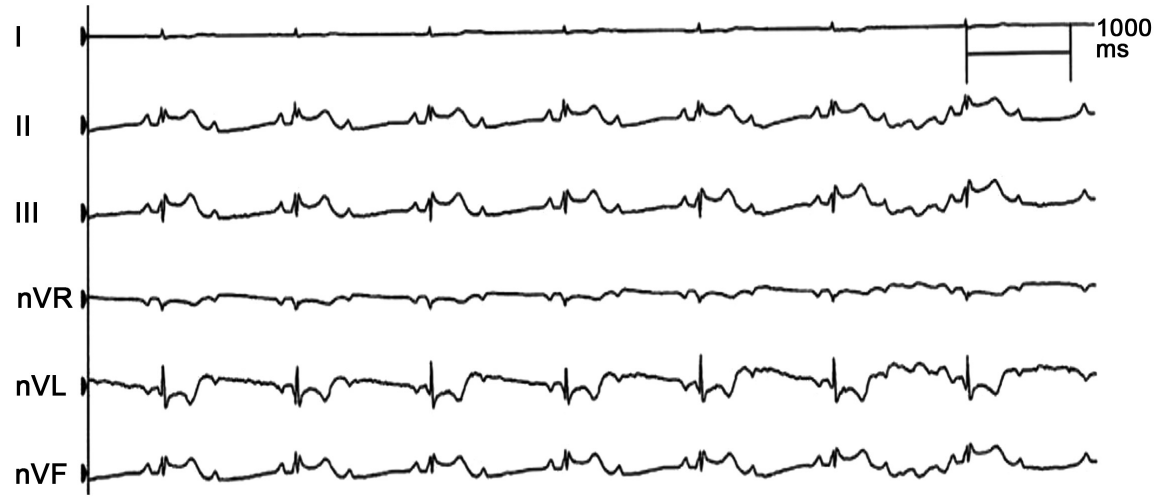

(a')

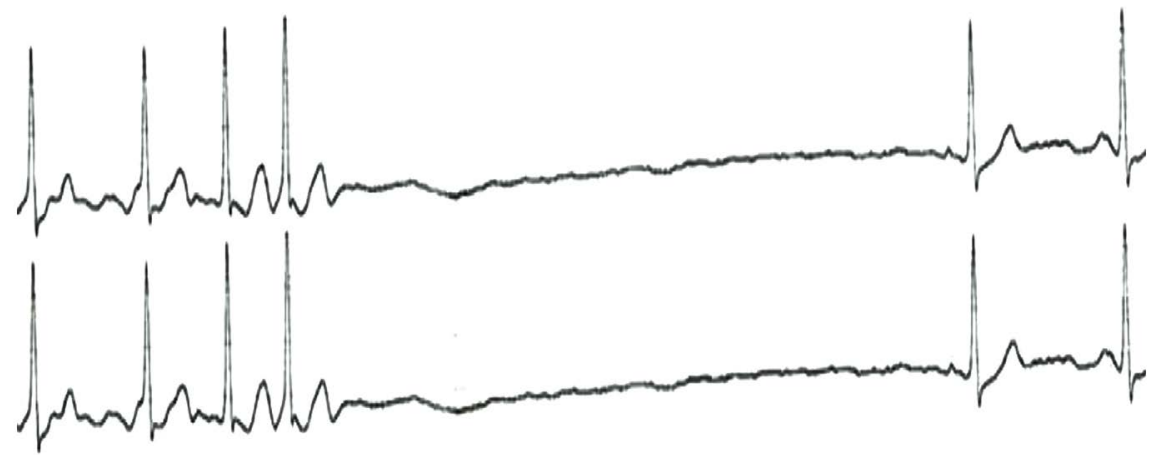

(b')

Figure 3. Second hospitalization, after transient ST elevation with chest pain during rehabilitation program. Provocation test. After 4 min occlusion of the distal segment of RCA1 (a) with high degree atrio-ventricular (AV) bloc (a'). At 5 minutes, occlusion of the proximal part (b) with asystole (b'). Normal RCA after $7 \mathrm{mg}$ of intracoronary Nitroglycerine (c).

images, may help to differentiate SCAD from atherosclerotic plaque disruption. The latter is known to be the major underlying mechanism of ACS. In our patient, the OCT run excluded both. Thus, prolonged severe coronary spasm leading to a thrombus formation may be one of the most plausible mechanisms explaining the clinical scenario despite the moderate atherosclerotic plaque found at the occlusion site. As previously reported by some IVUS characteristics of a spasm-induced vessel site series, minimal or moderate atherosclerosis is not uncommon [7] [8].

Pharmacological reperfusion was supported by STEMI presentation and long 
delay to the Cath lab. Nevertheless, one could argue that SCAD should have been taken into consideration before thrombolytic administration. In fact, fibrinolytic infusion as far as anticoagulant therapy should be avoided in SCAD patients since they can lead to severe complications [9].

We medically (double antiplatelet + low molecular weight heparin, statin, betablocker and nitrates) managed our patient given the importance of thrombus burden, the resolution of pain and ST segment elevation with TIMI 3 flow on angiogram. This attitude is closed to MIMI (minimalist immediate mechanical intervention) strategy [10].

Vasospastic angina is usually precipitated by hyperventilation and not exertion, despite the fact that the latter leads to hyperventilation. Provocative test has been realized to rule out spasm given the transient ST segment elevation with chest pain and due to the fact that the prior presentation was very suspicious of coronary spasm. The diagnosis was held according to COVADIS criteria [11]. Then the patient was put on Verapamil despite severe left ventricular ejection fraction impairment with close monitoring inward. Betablocker was removed definitely.

Aside from tobacco use, there was no history of cocaine or methamphetamines consumption in our patients. Depression, present in the history of our patient, is considered as an independent factor of occurrence of coronary spasm with a dose-effect relationship [12] and more generally of cardiovascular events [13]. Serious management of this aspect as well as of the other psycho-social factors is an integral part of the prevention of recurrences.

\section{Conclusion}

Vasospastic angina complicated by acute coronary syndrome can be challenging to diagnose. Intracoronary imaging can be helpful by elucidating ACS's underlying mechanism and may exclude classical diagnostic such as SCAD in young women naïve of traditional risk factors with an ambiguous angiogram.

\section{Conflicts of Interest}

The authors declare no conflicts of interest regarding the publication of this paper.

\section{References}

[1] Prinzmetal, M., Kennamer, R., Merliss, R., et al. (1959) Angina Pectoris. I. A Variant Form of Angina Pectoris; Preliminary Report. The American Journal of Medicine, 27, 375. https://doi.org/10.1016/0002-9343(59)90003-8

[2] Agewall, S., Beltrame, J.F., Reynolds, H.R., Niessner, A., Rosano, G., Caforio, A.L., De Caterina, R., Zimarino, M., Roffi, M., Kjeldsen, K., Atar, D., Kaski, J.C., Sechtem, U. and Tornvall, P. (2017) ESC Working Group Position Paper on Myocardial Infarction with Non-Obstructive Coronary Arteries. European Heart Journal, 38, 143-153. https://doi.org/10.1093/eurheartj/ehw149

[3] Oliva, P.B., Potts, D.E. and Pluss, R.G. (1973) Coronary Arterial Spasm in Prinzmetal 
Angina. Documentation by Coronary Arteriography. The New England Journal of Medicine, 288, 745-751. https://doi.org/10.1056/NEJM197304122881501

[4] Nakamura, M., Takeshita, A. and Nose, Y. (1987) Clinical Characteristics Associated with Myocardial Infarction, Arrhythmias, and Sudden Death in Patients with Vasospastic Angina. Circulation, 75, 1110-1116. https://doi.org/10.1161/01.CIR.75.6.1110

[5] Saw, J. (2014) Coronary Angiogram Classification of Spontaneous Coronary Artery Dissection. Catheterization and Cardiovascular Interventions, 84, 1115-1122. https://doi.org/10.1002/ccd.25293

[6] Motreff, P., Malcles, G., Combaret, N., Barber-Chamoux, N., Bouajila, S., Pereira, B., Amonchot, A., Citron, B., Lusson, J.-R., Eschalier, R. and Souteyrand, G. (2017) How and When to Suspect Spontaneous Coronary Artery Dissection: Novel Insights from a Single-Centre Series on Prevalence and Angiographic Appearance. Euro-Intervention, 12, e2236-e2243. https://doi.org/10.4244/EIJ-D-16-00187

[7] Yamagishi, M., Miyatake, K., Tamai, J., Nakatani, S., Koyama, J. and Nissen, S.E. (1994) Intravascular Ultrasound Detection of Atherosclerosis at the Site of Focal Vasospasm in Angiographically Normal or Minimally Narrowed Coronary Segments. Journal of the American College of Cardiology, 23, 352-357. https://doi.org/10.1016/0735-1097(94)90419-7

[8] Hong, M.K., Park, S.W., Lee, C.W., et al. (2000) Intravascular Ultrasound Findings of Negative Arterial Remodeling at Sites of Focal Coronary Spasm in Patients with Vasospastic Angina. American Heart Journal, 140, 395-401.

https://doi.org/10.1067/mhj.2000.108829

[9] Shamloo, B.K., Chintala, R.S., Nasur, A., Ghazvini, M., Shariat, P., Diggs, J.A., et al. (2010) Spontaneous Coronary Artery Dissection: Aggressive vs. Conservative Therapy. Journal of Invasive Cardiology, 22, 222-228.

[10] Belle, L., Motreff, P., Mangin, L., et al. (2016) Comparison of Immediate with Delayed Stenting Using the Minimalist Immediate Mechanical Intervention Approach in Acute ST-Segment-Elevation Myocardial Infarction: The MIMI Study. Circulation: Cardiovascular Interventions, 9, e003388. https://doi.org/10.1161/CIRCINTERVENTIONS.115.003388

[11] Beltrame, J.F., Crea, F., Kaski, J.C., et al. (2017) International Standardization of Diagnostic Criteria for Vasospastic Angina. European Heart Journal, 38, 2565-2568.

[12] Yoon, S.J., Kim, T.-S., Seung, K.B., Kim, P.J., Lee, C., Jun, T.-Y., et al. (2010) Role of Depressive Symptoms in Coronary Artery Spasm. Psychotherapy and Psychosomatics, 79, 191-193. https://doi.org/10.1159/000276379

[13] Daskalopoulou, M., George, J., Walters, K., Osborn, D.P., Batty, G.D., Stogiannis, D., et al. (2016) Depression as a Risk Factor for the Initial Presentation of Twelve Cardiac, Cerebrovascular, and Peripheral Arterial Diseases: Data Linkage Study of 1.9 Million Women and Men. PLoS ONE, 11, e0153838. https://doi.org/10.1371/journal.pone.0153838 